\title{
Pervivencias y estancamiento de una fortuna aristocrática en la Restauración. La Casa de Alcañices, 1869-1909
}

\author{
Juan Antonio Carmona Pidal *
}

El interés que ha despertado el estudio de la nobleza en la Europa contemporánea es aún relativamente reciente, aunque ya pone de relieve que lejos de desaparecer con el nacimiento de la sociedad burguesa mantuvo un fuerte peso hasta bien entrado el siglo Xx. El grupo nobiliario sin más reúne sin embargo dos grupos bien diferenciados, la vieja nobleza y la de nueva creación. La valoración indistinta de una y otra no da la medida de la trayectoria de ambas, al menos hasta que no hubiesen desaparecido las fronteras que las separaban. Las diferencias cobran todo su sentido cuando se estudian los patrimonios de una $u$ otra aristocracia en plena época isabelina, sobre todo por el peso que ocupa la tierra en las más tradicionales y su alejamiento de los sectores económicos más dinámicos, diferencias que parecen prorrogarse en los primeros años de la Restauración ${ }^{1}$. Resultado de este comportamiento económico es el declive económico general absoluto, pero sobre todo rela-

* Universidad Complutense.

1 Para un análisis cuantitativo, BaHAmONDE, A., "Crisis de la nobleza de cuna y consolidación burguesa (1840-1880)", en Madrid en la sociedad del siglo XIX, vol. 1, Madrid, 1984, págs. 326-375; GIMÉNEZ, Blanca, «La nobleza madrileña en el reinado de Alfonso XIl», Memoria de licenciatura, inédita. Universidad Complutense, 1986; CAYUELA, José Gregorio, "Encuesta sobre las fortunas de la élite madrileña en 1860 ». Memoria de licenciatura, inédita. Universidad Complutense, 1985. 
tivo, de esta vieja nobleza, como dejan ver las listas de contribuyentes ${ }^{2}$ y algún suceso de especial resonancia, como la muy conocida ruina del Duque de Osuna. La trayectoria económica de esta vieja y rica aristocracia es ya menos conocida a partir de la Restauración, especialmente desde los años 80 , en parte por un problema de fuentes, en parte también porque sus intereses parecen fundirse con los de esta nueva aristocracia decimonónica con intereses y fortunas mucho más diversificadas ${ }^{3}$. Los datos de que se disponen en la actualidad no permiten establecer aún modelos generales de comportamiento, ni de qué forma se fueron produciendo la convergencia entre estas antiguas y nuevas noblezas. De ahí la pertinencia de seguir profundizando en casos individuales, siempre en función de la disponibilidad e interés de los archivos particulares, sin duda la fuente que más claridad puede aportar en estos momentos al análisis de este grupo social.

La Casa de Alcañices ofrece pocos rasgos singulares dentro del grupo de los Grandes de España: los primeros marqueses habían fijado su residencia en Madrid en el siglo XVII y desde entonces habían ostentado con regularidad altos cargos palatinos, aunque debe destacarse que su influencia se habia vuelto más importante desde la época isabelina y el Sexenio; su fortuna, sin ser excepcional, ocupaba el 29 lugar de una lista de los mayores contribuyentes realizada en 1855 y el 44 en $1875^{4}$.

En 1868 fallecía Nicolás Osorio y Zayas, XIV Marqués de Alcañices, Marqués de los Balbases, duque de Algete y de Alburquerque, cuatro veces grande de España y con otros catorce títulos más. Le había tocado vivir, durante los cuarenta años que estuvo al frente del patrimonio, numerosos años de crisis y los cambios legales, sociales y económicos que acompañaron la Revolución Liberal de la que sería un ardiente defensor. Su patrimonio no sufrirá por ello. Dejaba 36.500 hectáreas de tierras repartidas por toda la península, que producían cerca de 2.500 .000 reales anuales ${ }^{5}$. Estas cifras eran de hecho superiores a las que había disfrutado su familia a fines del siglo XVIII, prueba fehaciente de que la Casa había pasado con éxito la Revolución Liberal. Sin embargo, las causas

2 Congost, Rosa, "Las listas de los mayores contribuyentes de 1875", Agricultura y sociedad, núm. 27, (1983).

${ }^{3}$ Con mucho, el obstáculo más decisivo es el secreto notarial, dado que impide (sin previa autorización de la familia implicada) el uso de fuentes notariales más allá de 1889 .

${ }^{4}$ Congost, Rosa, "Las listas de los mayores contribuyentes de 1875 ", Agricultura y sociedad, núm. 27, (1983).

"Archivo Alburquerque, "Memoria estadística", 1857; Archivo Histórico de Protocolos Notariales de Madrid, protocolos $27.680-2$ y $27.688-90$. 
deben verse en una combinación de factores en buena parte externos a la capacidad de gestión del Marqués de Alcañices. Por un lado conviene destacar la estructura de la fortuna heredada a finales del siglo XVIII, de naturaleza territorial en su gran mayoría (en un 65 por 100) y poco sensible en consecuencia a los conflictos inherentes a la abolición de los señoríos. Por otro, se hallaba relativamente limpio de deudas gracias a una labor de medio siglo de amortizaciones (1740-1790). Con este patrimonio, la Casa tuvo que enfrentarse a medio siglo de crisis (1800-1845/ 50 ), producida por la descomposición del antiguo régimen. La crisis se definía en primer lugar como una crisis de rentas, esencialmente de las señoriales, pero también de las territoriales a consecuencia de la depresión agraria iniciada poco después del final de la guerra de la Independencia. Pero era sobre todo una crisis financiera, provocada en parte por el descenso de las rentas y, sobre todo, por una ruptura de los canales crediticios tradicionales que le impedían satisfacer su nivel tradicional de consumo. La desaparición del censo y el mantenimiento del mayorazgo bloquearon virtualmente la Casa, impidiéndole el acceso al mercado crediticio. La consecuencia de este fenómeno radicaba más en una reducción del gasto (incluido por ejemplo, el retraso de la boda del primogénito) que la espiral de endeudamiento que habian protagonizado sus antepasados en el siglo XVII.

El recurso al crédito o la enajenación patrimonial fue por tanto bien breve, de 1834 a 1845/1850 (coincidiendo con un aumento del gasto general) y no alcanzó a poner en peligro la salud económica del patrimonio ${ }^{6}$. El comienzo de la Década Moderada señalaría también la normalización de muchas situaciones dudosas: se indemnizaron los diezmos con más de ocho millones de reales en Deuda pública (a partir de $1850^{7}$ ), la Hacienda Pública se hacía cargo provisionalmente de las alcabalas y otras rentas enajenadas ofreciéndole una renta anual de 300.000 reales, mientras se sucedian las victorias en los pleitos sobre señoríos ${ }^{8}$. Pero mucho más decisivo fue para la Casa el inicio de una coyuntura excepcional de precios agrarios en crecimiento (a partir de 1840/50). El sistema tradicional de arrendamientos a corto plazo resultó

\footnotetext{
${ }^{6}$ Aunque el servicio de la deuda exigiese en torno al 40 por 100 de la renta neta aproximadamente.

7 Archivo Alburquerque, "Memoria estadística», 1857; Archivo Histórico de Protocolos Notariales de Madrid, protocolos 27.680-2 y 27.688-90.

${ }^{8}$ El más notable y de más larga duración enfrentaría a la Casa con los cincuenta y cuatro pueblos que componían el propio marquesado de Alcañices desde 1815 a 1845 (Archivo Histórico Nacional, leg. 32.246). También constan los del marquesado de Cuéllar, y las villas de Grajal, Fuensaldaña, Almanza, Ayoo por citar sólo las más importantes.
} 
ser sumamente eficaz, como lo atestigua el doblamiento de la renta en ese escaso período de tiempo y que del mismo modo ponía en evidencia la prevalencia del terrateniente en el agro español. Por otra parte esta coyuntura excepcional le ofreció la oportunidad de incrementar sus gastos corrientes (pero no las inversiones) manteniendo los balances equilibrados e incluso reducir el peso del servicio de la deuda (que seguia siendo de 8 millones) sin necesidad de amortizarla ${ }^{9}$.

A su muerte la Casa disponía de un patrimonio muy revalorizado con respecto a 20 años antes y relativamente menos endeudado, aunque su estructura se mantenía muy alejada a la de cualquier gran burgués madrileño recién ennoblecido ${ }^{10}$. Sin embargo con la práctica del mayorazgo encubierto, lograba transmitir el patrimonio, casi intacto, a su primogénito.

José Osorio y Silva (1825-1909) tenía ya 43 años en el momento de hacerse cargo del patrimonio, pero no era ningún desconocido en el mundo político. Había desempeñado ya con el título de marqués de Cuéllar varias actas de diputado desde 1851 y, ya con recién rehabilitado título de duque de Sesto, de los cargos de alcalde y de gobernador civil de Madrid, amén de varios puesto palatinos. Pero será realmente en el Sexenio cuando adquiera una posición política que pocos nobles de cuna compartirían a lo largo de todo el siglo. Como ayo del Príncipe Alfonso tuvo una gran influencia en la compleja trama que supuso la formación del partido alfonsino. Su papel no se limitó al político. Aportó considerables sumas de dinero para el mantenimiento de la familia real en el exilio y la financiación de la Restauración ${ }^{11}$. Simultáneamente abriría los salones que sus padres habian mantenido relativamente cerrados hasta hacer de ellos el mayor centro social de la vieja y nueva aristocracia durante el Sexenio. Con la Restauración el marqués ostentaría el máximo cargo palatino, el de jefe de Palacio, hasta 1885, lo que le obligaría asimismo a conservar el brillo social otros diez años más.

El protagonismo poítico del ahora marqués de Alcañices no fue gratuito. El propio José Osorio pudo ser consciente desde el primer mo-

${ }^{9}$ No debe olvidarse que el patrimonio era dinámico y su crecimiento se debía a la nueva revalorización de activos. Ello coadyuvó a un saneamiento indirecto en consonancia con la protagonizaría otros nobles. La eficacia del saneamiento emprendido por los Duques de Medinaceli se debía, quizás, más a esta expansión agricola que a una hábil reestructuración; BAHAMODE, op. cit., págs. 333 y ss.

10 Varios casos en BaHAMONDE, Op. cit., págs. 364 y ss.

${ }^{11}$ Espadas Burgos, Manuel, Alfonso XII y los origenes de la Restauración, Madrid, 1975, págs. $249-262$. 
mento que supondría el sacrificio de una parte de su herencia, dado que las rentas corrientes no alcanzarían nunca a satisfacer los desembolsos debidos a la causa y a la "sobrerepresentación», esto es, el mantenimiento de un tren de vida por encima de los niveles de renta habituales. El gasto total, sin desglosar, alcanzó una suma impresionante, probablemente entre 38 y 44 millones de reales, una verdadera fortuna consumida en apenas 16 años, o sea de 2 a 2,5 millones anuales, lo que le permitía doblar sus rentas corrientes. Es evidente que apenas realizada la operación de testamentaría que había evaluado la fortuna heredada en 57 millones, el duque era perfectamente consciente de que estaba dilapidando su herencia y arriesgando su futuro económico. La "empresa», si puede llamarse así la apuesta por la restauración alfonsina y la transformación de su Casa en un símbolo de la aristocracia renovada y socialmente brillante, fue todo un éxito - desde un punto de vista social- aunque, económicamente, fuera una empresa sin compensaciones.

Los recursos eran suministrados a través de tres canales diferentes, pero ya clásicos: el recurso al pagaré, a la hipoteca y finalmente a la venta. El recurso al crédito en forma de pagarés presentaba dos obstáculos que empezaron a hacer mella durante el Sexenio: la existencia de una importante deuda a corto plazo heredada de su padre, y no sostenible sin garantías, y la propia situación revolucionaria que convertía a la nobleza en deudora de poca confianza. El resultado no podía ser menos que un incremento de las tasas de interés hasta el 8 y el 10 por 100 y el recurso a la hipoteca desde 1870. Muy poco después, y coincidiendo con el máximo de gastos (1871-74) se recurrió simultáneamente a los tres sistemas. El proceso no concluyó con la llegada al trono de Alfonso XII. Todo hace pensar que el marqués, nuevo jefe de Palacio, no logró sacar provecho de su nueva situación, y mantuvo por su cuenta un tren de gasto apropiado a su status y el de su mujer, pero que no correspondía realmente al estado de su fortuna y de sus numerosas deudas. En 1873 había culminado el proceso de liquidación de su mayor administración, la de Salamanca, y en 1881 procedía a deshipotecar su gran mansión, dispuesto por tanto a enajenarla y construirse otra en un solar recién adquirido en las afueras. Sin embargo, el palacio nunca llegaría a construirse.

El impacto económico de estos años fue necesariamente importante, aunque paradójicamente no alteró en su esencia el funcionamiento de la Casa. El marqués asumió la reducción de sus rentas y la precariedad financiera de su administración sin realizar cambios de envergadura. Es verdad que tampoco llegó a arruinarse. 
Después de 1885, se pueden distinguir dos etapas en la marcha de la economía que separa el año 1896, punto de inflexión de los peores momentos económicos. Hasta ese momento, al alto pasivo (en torno a 10 millones de reales) se le sumaban los efectos de la Gran Depresión (a partir de 1883/4) dada la sensibilidad del patrimonio a la evolución de los precios agrícolas. Ello no fue un obstáculo para que la Casa lograra cierto equilibrio, de hecho hay indicios de que la deuda se mantuvo relativamente estable, una consecuencia por tanto de una reducción más que proporcional de sus gastos ${ }^{12}$.

Desde el punto de vista de la renta, los efectos de la Depresión no repercutieron de modo indiscriminado en el patrimonio agrario: fueron más sentidos en las dos mil pequeñas explotaciones de secano y en las rentas de origen señorial: retrasos en el cobro de las rentas, las resistencias a su pago, las reiteradas condonaciones o perdón de rentas parecen ser el motivo de que el marqués decidiera acabar de liquidar las administraciones con predominio de pequeñas explotaciones ${ }^{13}$, aprovechando la devolución de un importante préstamo de 2.400 .000 rsv. a favor de la marquesa viuda de Comillas. Pero la racionalización se extendió también al propio sistema administrativo, con el abandono de prácticas tradicionales en el reclutamiento del personal administrativo, la exigencia de un número cada vez mayor de personal especializado y, finalmente, el empleo de ingenieros agrónomos a la hora de realizar planes agrícolas. En Cadreíta (Navarra) se empiezan a producir nuevos cultivos como la remolacha, la patata o el maíz. En Cuéllar (Segovia) la recogida de resina de sus numerosos pinares se encomienda a una empresa, la Unión Resinera, cuyo director asesoraría directamente el cultivo de los pinares. Se observa la ampliación del cultivo directo (aunque seguía limitado a Cadreíta y la finca de recreo de Algete) e incluso la aparición de los primeros contratos de aparcería. Pero no se puede imputar realmente a estas transformaciones la rápida recuperación de la renta a la que se asiste después de 1896 dado que el crecimiento de la renta afectó por igual a las administraciones donde se habían cometido las reformas y las que permanecieron bajo el mismo sistema de explotación como Noez (Toledo) o Lebrija (Sevilla). Asimismo, los cambios operados en Algete o Cuéllar no impidieron que la primera siguiera siendo deficitaria y la se-

12 No es muy seguro de todas formas, que esa austeridad fuera tan voluntaria y obedeciera al deseo de ver sus finanzas equilibradas: la pérdida de sus cargos palatinos (a partir de 1885), su elevada edad e incluso el fallecimiento de su esposa, la Princesa de Troubetzkoy, en 1896, pudo haber coadyuvado a esa reducción.

${ }^{13}$ Se trataba de las administraciones de Grajal, Palazuelo, Huelma y Almanza. 
gunda no recuperara las rentas de 1866. Un fenómeno que asienta el peso que aún desempeñaba las rentas de la tierra en la agricultura española de comienzos de siglo y probablemente principal beneficiaria de la política proteccionista.

La mejoría de la coyuntura no significó sin embargo, una verdadera reconversión patrimonial. No se asiste por ejemplo, a un verdadero proceso de saneamiento que se hubiera traducido en una reducción del pasivo. Podemos creer que pudo obedecer a dos motivos: el alza de las rentas combinada con un descenso de los tipos de interés beneficiaba en un primer momento al deudor haciendo poco rentable una amortización del pasivo a costa de un activo en curso de revalorización ${ }^{14}$. El otro motivo obedece más a actitudes mentales: la amortización de la deuda habría supuesto literalmente la reducción del patrimonio a la mitad (en el número de estados, por ejemplo, de los que apenas conservaba seis) empobreciendo aún más a esta familia con perjuicio de su status, en una sociedad cada vez más impregnada de valores materiales ${ }^{15}$. De esta forma el pasivo absorbía cerca del 52 por 100 de la renta neta en 19121914 , signo de que la fortuna estaba lejos de hallarse libre de dificultades.

El marqués de Alcañices, decano de la Grandeza de España, morirá en 1909 a los 84 años de edad, dejando en los albores del siglo Xx una fortuna de estructura muy similar a la que heredaría de su padre $o$ incluso a la de su abuelo 100 años antes ${ }^{16}$. Agraria en su inmensa mayoría, seguía percibiendo unas rentas que eran vestigios de su pasado señorial (en Alcañices por ejemplo) y aún seguía pendiente de que se le indemnizara sus derechos sobre la Albufera. Más llamativa es la total ausencia de valores industriales, bancarios o públicos (por debajo incluso que su padre), lo que probablemente le alejaba aún más de las estructuras de fortunas contemporáneas, precisamente cuando la seguridad deja de ser un atributo exclusivo de las rentas agrarias. La crisis y las transformaciones económicas no parecieron haber modificado en definitiva la actitud de este gran propietario hacia la tierra y sus recelos hacia los valores industriales. Unas pocas compras realizadas en Algete y Al-

\footnotetext{
${ }^{14}$ La inflación de la Primera Guerra Mundial les sería aún más favorable. Para el caso inglés, StONe, Lawrence, An Open Elite? England, 1540-1880, Oxford, 1984, pág. 12.

${ }_{15}$ CANNADINE, David, "Aristocratic Indebtedness in the Nineteenth Century: The Case Re-Opened", Economic History Review, 30, (1977), págs. 638; Stone, L., Op. cit., pág. 13.

${ }^{16}$ Archivo de Alburquerque, Inventario de JOSÉ OSORIO Y SILvA, José, 1909.
} 
cañices (en su mayoría montes de poco valor pero mucha extensión ${ }^{7}$ ), y con escasos resultados económicos explicitan su desinterés por los valores mobiliarios.

Sin embargo, el balance económico es sólo ligeramente negativo: las ventas se paralizan prácticamente en 1896, sus rentas crecen a partir de esta fecha y logra conservar una parte importante de su gasto tradicional: unos 15 criados, varios coches, los numerosos caballos instalados en el propio casco urbano, su pequeño palacio, la finca de recreo, etc... La Casa sigue siendo además una de las mayores propietarias del país, la decimosexta en 1932 según la encuesta del Instituto de Reforma Agraria ${ }^{18}$. Estos éxitos difícilmente pueden atribuirse al espíritu emprendedor de José Osorio que prefirió volcar su sentido de la iniciativa y del riesgo en una acción política, como lo hicieran sus antepasados.

De hecho la administración conserva los resortes de una economía del gasto, atemperados, es verdad, por un prudente ajuste de los gastos en los momentos críticos, pero sin el ahorro imprescindible para llevar a cabo estrategias expansivas; pervivencias que se apoyan sin duda en la propia estabilidad y solidez de la renta.

${ }^{17}$ Como dos montes adquiridos a finales del siglo $x \mid x, y$ cuyas 1.300 hectáreas eran valoradas en menos de 60.000 reales en el inventario.

18 «Relación rectificada de la superficie declarada a los efectos de la Reforma agraria, por cada uno de los 99 individuos de la extinguida grandeza de España que habiendo ejercido sus prerrogativas honoríficas poseen fincas en el territorio nacional", Boletín del Instituto de Reforma Agraria, 3, 25 (julio, 1934), págs. 539-43. 